\title{
A NEW SPARSITY-ENABLED SIGNAL SEPARATION METHOD BASED ON SIGNAL RESONANCE
}

\author{
Ivan W. Selesnick \\ Electrical and Computer Engineering \\ Polytechnic Institute of New York University \\ Brooklyn, NY 07040 \\ selesi@poly.edu
}

\begin{abstract}
This paper proposes the separation of signal components based on resonance. The method relies on several recent developments in sparse signal processing: morphological component analysis (MCA), the rational-dilation wavelet transform (RADWT), and fast algorithms for $\ell_{1}$-norm regularized linear inverse problems (for example, SALSA). The RADWT allows one to extract signal components according to resonance characteristics because the RADWT allows the Q-factor (frequency resolution) of the wavelet transform to be varied. The sought decomposition can not be accomplished by frequency-based filtering. An example illustrates the method.
\end{abstract}

Index Terms - Sparse signal representation, wavelets, morphological component analysis, Q-factor.

\section{INTRODUCTION}

The decomposition of a signal into a set of frequency components, as achieved by a suitably designed filter bank or by the Fourier transform, is a fundamental conceptual and computational tool in signal processing. Of course, many natural signals are more accurately and efficiently described as a sum of finite-duration (or windowed) frequency components; and for such signals time-frequency representations like the short-time Fourier transform (STFT) and wavelet transforms (WTs) are often more appropriate.

However, many signals contain oscillatory components that are not well captured by either a single STFT or a single WT. For example, a given signal may contain multiple oscillatory pulse-like components with a common oscillation frequency $f_{o} \mathrm{~Hz}$, where each oscillatory pulse is of different duration - that is, each oscillatory component may comprise a different number of cycles. In the terminology of filters, each oscillatory pulse may have a different Q-factor (quality-factor). Figure 1 illustrates a set of pulses with various frequencies and Q-factors. Pulses 1 and 2 have the same oscillation frequency, yet they have a different number of cycles.

Given a single signal consisting of all six pulses illustrated in Fig. 1, where the pulses overlap in time, conventional frequency and time-frequency representations will not be able to decompose the signal into its separate pulses. These representations will be able to separate pulses 1 and 2 from pulses 3 and 4 because pulses 1 and 2 are of a different frequency than pulses 3 and 4 . But pulses 1 and 2 can not be separated using conventional linear time-frequency signal transforms like the STFT or WTs, because these two pulses are of the same frequency and may overlap in time. On the other hand, pulses 1 and 2 do have substantially different Q-factors (resonance).
In this paper, we propose the decomposition of a signal into components based, not on frequency, but on resonance. The proposed method is designed to separate pulses 1 and 2 in Fig. 1 for example, even if they overlap in time. This provides an alternative approach to the separation (analysis) of oscillatory signals, such as EEG and other physiological signals, and vibration signals arising in physical sciences, etc.

The proposed resonance-based signal separation method is based upon several recent developments in signal processing: morphological component analysis (MCA) [17], new rationaldilation wavelet transforms [1], and new fast algorithms for sparseregularized linear inverse problems [12]. These developments, each of which advances sparsity-based signal processing, will be reviewed in Section 2.

The signal separation problem posed in this paper (based on resonance) is closely related to the problem of decomposing a signal into transient and oscillatory (or tonal) components, which has been addressed by several authors $[6,7,10,14,15]$. In [14] the goal is efficient audio compression, and it is proposed that sinusoidal components, transients, and a noise-like residual be separately coded. The separation of transient and tonal components using sparse signal representations is developed in $[6,7,15]$. The wavelet transform and the modified discrete cosine transform (MDCT) are used in [6,15], while overcomplete modulated complex lapped transform (MCLT) dictionaries are utilized in [7]. A general Bayesian approach is described in [10] and is illustrated using an MDCT basis with a long time window for the tonal component and an MDCT with a short time window for the transient component. An example of tonal/transient separation in audio is also given in [13] which introduces the timefrequency jigsaw puzzle. An alternative novel approach to transient separation is the time-scale method introduced in [4], which exploits the multi-scale characterization of transients.

Some of the ideas in this paper were presented earlier in [16] for the problem of transient/oscillatory component separation.

\section{SPARSE REPRESENTATIONS}

\subsection{Rational-dilation wavelet transform (RADWT)}

The dyadic discrete wavelet transform (DWT) is well-known to enable the sparse representation of piecewise smooth signals. However, the sparse representation of oscillatory signals requires transforms with higher frequency resolution than the dyadic DWT. In order to address this need, a new family of (overcomplete) wavelet transforms were developed in [1] for which one has the ability to tune the Q-factor of the analysis/synthesis functions. Instead of be- 
oscillatory pulse

1

2

3

4

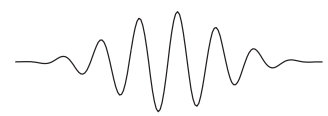

5

6

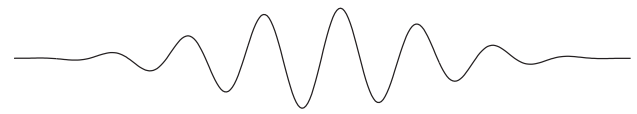

frequency

duration

$f_{o}$

$f_{o}$

$f_{o} / 2$

$2 T$

$f_{o} / 2$

$8 T$

$f_{o} / 4$

$4 T$

$f_{o} / 4$

$16 T$

Fig. 1. Oscillatory pulses of various frequencies and durations. Pulses 1, 3, and 5 have the same (low) Q-factor; these oscillatory pulses have essentially a single cycle; they are of low resonance. Pulses 2, 4, and 6 have the same (higher) Q-factor; these oscillatory pulses have more cycles and are of a higher resonance. On the other hand, pulses 1 and 2 have the same frequency; etc. A low Q-factor wavelet transform (the classical dyadic wavelet transform) is suitable for the efficient representation of pulses 1, 3, and 5. The efficient representation of pulses 2, 4, and 6 calls for a wavelet transform with higher Q-factor. Each illustrated pulse is a sine wave windowed with a Blackman window.

ing based on integer dilations, this family of WTs are based on rational dilations with dilations factors between 1 and 2 . Setting the dilation factor close to 1 gives a WT with analysis/synthesis functions (wavelets) having a high Q-factor. Setting the dilation factor close to (or equal to) 2 gives a WT with the same low Q-factor as the dyadic DWT. The wavelet transforms introduced in [1] are modestly redundant and have the perfect reconstruction property. Specifically, the WTs are self-inverting (the synthesis filters for perfect reconstruction are the time-reversed analysis filters).

Few papers have addressed the design and application of rational-dilation wavelet transforms, likely because multiresolution analysis theory, and the design of paraunitary FIR filter banks, is substantially more difficult for the rational-dilation case than for the integer-dilation case. However, as demonstrated in [1], if the transform is allowed to be overcomplete and the filters are allowed to have non-rational transfer functions, then an iterated filter bank can be readily designed for the implementation of a rationaldilation WT (RADWT). Even though the filter bank is implemented in [1] using the FFT, the basis functions are well localized in the time-frequency plane and highly regular due to the design of the frequency responses.

\subsection{Morphological component analysis (MCA)}

Morphological component analysis (MCA) is a general approach to decompose a signal into two or more components based on the sparse representation of the signal components in respective transforms.
In order for MCA to work, the respective transforms must have a low coherence (the analysis/synthesis functions of each transform should have low correlation with the analysis/synthesis functions of the other transforms). MCA is introduced and elaborated in a series of papers, including Refs. [9, 17, 18].

Given an observed signal $\mathbf{x}$ that is the superposition of two signals,

$$
\mathbf{x}=\mathbf{x}_{1}+\mathbf{x}_{2}, \quad \mathbf{x}, \mathbf{x}_{1}, \mathbf{x}_{2} \in \mathbb{R}^{N},
$$

the goal of MCA is to estimate/determine $\mathbf{x}_{1}$ and $\mathbf{x}_{2}$ individually. MCA assumes that $\mathbf{x}_{1}$ and $\mathbf{x}_{2}$ can be sparsely represented in bases (or frames) $\mathbf{S}_{1}$ and $\mathbf{S}_{2}$ respectively. The MCA approach, in one form (MCA can be formulated in different ways), asks that the objective function,

$$
J\left(\mathbf{w}_{1}, \mathbf{w}_{2}\right)=\left\|\mathbf{x}-\mathbf{S}_{1} \mathbf{w}_{1}-\mathbf{S}_{2} \mathbf{w}_{2}\right\|_{2}^{2}+\lambda_{1}\left\|\mathbf{w}_{1}\right\|_{1}+\lambda_{2}\left\|\mathbf{w}_{2}\right\|_{1}
$$

be minimized with respect to $\mathbf{w}_{1}$ and $\mathbf{w}_{2}$. We use the $\ell_{1}$-norm here because it promotes sparsity yet is a convex function. Then components, $\hat{\mathbf{x}}_{1}$ and $\hat{\mathbf{x}}_{2}$, estimated by MCA are

$$
\hat{\mathbf{x}}_{1}=\mathbf{S}_{1} \mathbf{w}_{1 *}, \quad \hat{\mathbf{x}}_{2}=\mathbf{S}_{2} \mathbf{w}_{2 *},
$$

where $\left(\mathbf{w}_{1 *}, \mathbf{w}_{2 *}\right)$ minimizes the objective function (1).

More general forms of MCA allow the sparsity measures for $\mathbf{x}_{1}$ and $\mathbf{x}_{2}$ in (1) to be different from each other; additionally, the data fidelity term need not be an $\ell_{2}$ norm. Additionally, other sparsity priors besides the $\ell_{1}$-norm can be used, etc. 
MCA has been demonstrated to be very effective for image inpainting and other image processing problems, especially with the curvelet transform, the block 2D DCT, and 2D wavelet transforms in the role of $\mathbf{S}_{1}$ and $\mathbf{S}_{2}[9,17,18]$.

For multi-resonance signal separation, as proposed in Section 1, we suggest that the rational-dilation wavelet transform (RADWT) be used for sparse signal representations in MCA. By using two or more RADWTs with differing Q-factors, we propose that components of a signal having different resonance characteristics can be separated from one another.

\subsection{Split augmented Lagrangian shrinkage algorithm (SALSA)}

The use of MCA, as formulated here, requires the minimization of the objective function (1). Although $J$ is convex, the nondifferentiability of the $\ell_{1}$-norm can make the minimization difficult; and many algorithms have recently been proposed to minimize this type of objective function. An important early algorithm is the iterated soft-thresholding algorithm (ISTA) developed in [5, 11]. However, many faster algorithms have since been developed; a few examples are Refs. [2,3, 8, 19].

A particularly fast recent algorithm for a class of $\ell_{1}$-regularized linear inverse problems is the 'split augmented Lagrangian shrinkage algorithm' (SALSA) developed in [12]. Each iteration of SALSA calls for the solution of an $\ell_{2}$-regularized inverse problem, which may itself be a challenge. However, for numerous problems including the one considered here, the relevant $\ell_{2}$ problem can be written essentially in closed form.

In order to specialize SALSA to the MCA problem (1), define

$$
\mathbf{H}=\left[\begin{array}{ll}
\mathbf{S}_{1} & \mathbf{S}_{2}
\end{array}\right], \quad \mathbf{u}=\left[\begin{array}{l}
\mathbf{u}_{1} \\
\mathbf{u}_{2}
\end{array}\right], \quad \mathbf{w}=\left[\begin{array}{l}
\mathbf{w}_{1} \\
\mathbf{w}_{2}
\end{array}\right] .
$$

Then minimizing $J$ in (1) via SALSA gives the iterative algorithm:

$$
\begin{gathered}
\mathbf{u}^{(k+1)}=\arg \min _{\mathbf{u}}\|\mathbf{y}-\mathbf{H} \mathbf{u}\|_{2}^{2}+\mu\left\|\mathbf{u}-\mathbf{w}^{(k)}-\mathbf{d}^{(k)}\right\|_{2}^{2} \\
\mathbf{w}^{(k+1)=} \arg \min _{\mathbf{w}} \lambda_{1}\left\|\mathbf{w}_{1}\right\|_{1}+\lambda_{2}\left\|\mathbf{w}_{2}\right\|_{1} \\
\quad+\mu\left\|\mathbf{u}^{(k+1)}-\mathbf{w}-\mathbf{d}^{(k)}\right\|_{2}^{2} \\
\mathbf{d}^{(k+1)}=\mathbf{d}^{(k)}-\mathbf{u}^{(k+1)}+\mathbf{w}^{(k+1)}
\end{gathered}
$$

where $k$ is the index of iteration. The parameter $\mu$ needs to be selected by the user. See [12] for details. We have used $\mu=0.5 \lambda$ in the MCA experiments below.

Note that (3) is an $\ell_{2}$ problem and therefore the minimization in (3) can be expressed straightforwardly:

$$
\mathbf{u}^{(k+1)}=\left(\mathbf{H}^{t} \mathbf{H}+\mu \mathbf{I}\right)^{-1}\left(\mathbf{H}^{t} \mathbf{y}+\mu\left(\mathbf{w}^{(k)}+\mathbf{d}^{(k)}\right)\right) .
$$

Using $\mathbf{S}_{1} \mathbf{S}_{1}^{t}=\mathbf{I}$ and $\mathbf{S}_{2} \mathbf{S}_{2}^{t}=\mathbf{I}$ (which is valid for the RADWT of [1] because it is self-inverting) and the matrix inverse lemma, we can write:

$$
\left(\mathbf{H}^{t} \mathbf{H}+\mu \mathbf{I}\right)^{-1}=\frac{1}{\mu} \mathbf{I}-\frac{1}{\mu(\mu+2)}\left[\begin{array}{l}
\mathbf{S}_{1}^{t} \\
\mathbf{S}_{2}^{t}
\end{array}\right]\left[\begin{array}{ll}
\mathbf{S}_{1} & \mathbf{S}_{2}
\end{array}\right] .
$$

Also, note that (4) is an $\ell_{1}$-norm regularized denoising problem and therefore the minimization in (4) is given by soft-thresholding.
Therefore, SALSA for the MCA problem as formulated here is:

$$
\begin{aligned}
\mathbf{b}_{1}^{(k)} & =\mathbf{S}_{1}^{t} \mathbf{y}+\mu\left(\mathbf{w}_{1}^{(k)}+\mathbf{d}_{1}^{(k)}\right) \\
\mathbf{b}_{2}^{(k)} & =\mathbf{S}_{2}^{t} \mathbf{y}+\mu\left(\mathbf{w}_{2}^{(k)}+\mathbf{d}_{2}^{(k)}\right) \\
\mathbf{c}^{(k)} & =\mathbf{S}_{1} \mathbf{b}_{1}^{(k)}+\mathbf{S}_{2} \mathbf{b}_{2}^{(k)} \\
\mathbf{u}_{1}^{(k+1)} & =\frac{1}{\mu} \mathbf{b}_{1}^{(k)}-\frac{1}{\mu(\mu+2)} \mathbf{S}_{1}^{t} \mathbf{c}^{(k)} \\
\mathbf{u}_{2}^{(k+1)} & =\frac{1}{\mu} \mathbf{b}_{2}^{(k)}-\frac{1}{\mu(\mu+2)} \mathbf{S}_{2}^{t} \mathbf{c}^{(k)} \\
\mathbf{w}_{1}^{(k+1)} & =\operatorname{soft}\left(\mathbf{u}_{1}^{(k+1)}-\mathbf{d}_{1}^{(k)}, \frac{\lambda_{1}}{2 \mu}\right) \\
\mathbf{w}_{2}^{(k+1)} & =\operatorname{soft}\left(\mathbf{u}_{2}^{(k+1)}-\mathbf{d}_{2}^{(k)}, \frac{\lambda_{2}}{2 \mu}\right)
\end{aligned}
$$

where $\operatorname{soft}(x, T)$ is the soft-threshold rule with threshold $T$.

\section{EXAMPLES}

To illustrate the proposed resonance-based signal separation method, a synthetic test signal was created, illustrated in Fig. 2. The test signal consists of six pulses of three different frequencies and two different Q-factors (as in Fig.1). The goal is to separate the signal into a high and a low resonance component. Applying 100 iterations of SALSA, as illustrated in the figure, yields a signal separation according to resonance, as desired. In this example, we used $\lambda_{1}=\lambda_{2}=0.2$ and RADWTs with dilation factors $6 / 5$ and $3 / 2$, each with a redundancy of 3 .

Note that frequency domain filtering will be unable to achieve the desired separation. For example, high-pass filtering will extract the high and low resonance pulses of high frequency.

We also applied 100 iterations of ISTA. The result (not shown) is inferior because ISTA requires many more iterations to converge than SALSA. (To clarify - ISTA will minimize the objective function if allowed to run for more iterations.) The decay of the objective function (1) is illustrated in Fig. 3 for both ISTA and SALSA.

\section{CONCLUSION}

This paper proposes a resonance-based signal separation method that relies on techniques for sparse signal representations. MCA provides the framework for the method in which a sparse representation is sought for each signal component. The method utilizes a rational-dilation wavelet transform (RADWT) for the sparse representation of each resonance component. The RADWT allows one to extract signal components according to their resonance characteristics. Moreover, the RADWT is a self-inverting fully-discrete transform which is important for the SALSA algorithm as described.

Under current investigation is the application of the developed resonance-based signal separation to biomedical signal analysis, speech and audio processing, and as a preprocessing step for shorttime spectral estimation of non-stationary signals.

\section{REFERENCES}

[1] İ. Bayram and I. W. Selesnick. Frequency-domain design of overcomplete rational-dilation wavelet transforms. IEEE Trans. on Signal Processing, 57(8):2957-2972, August 2009. 

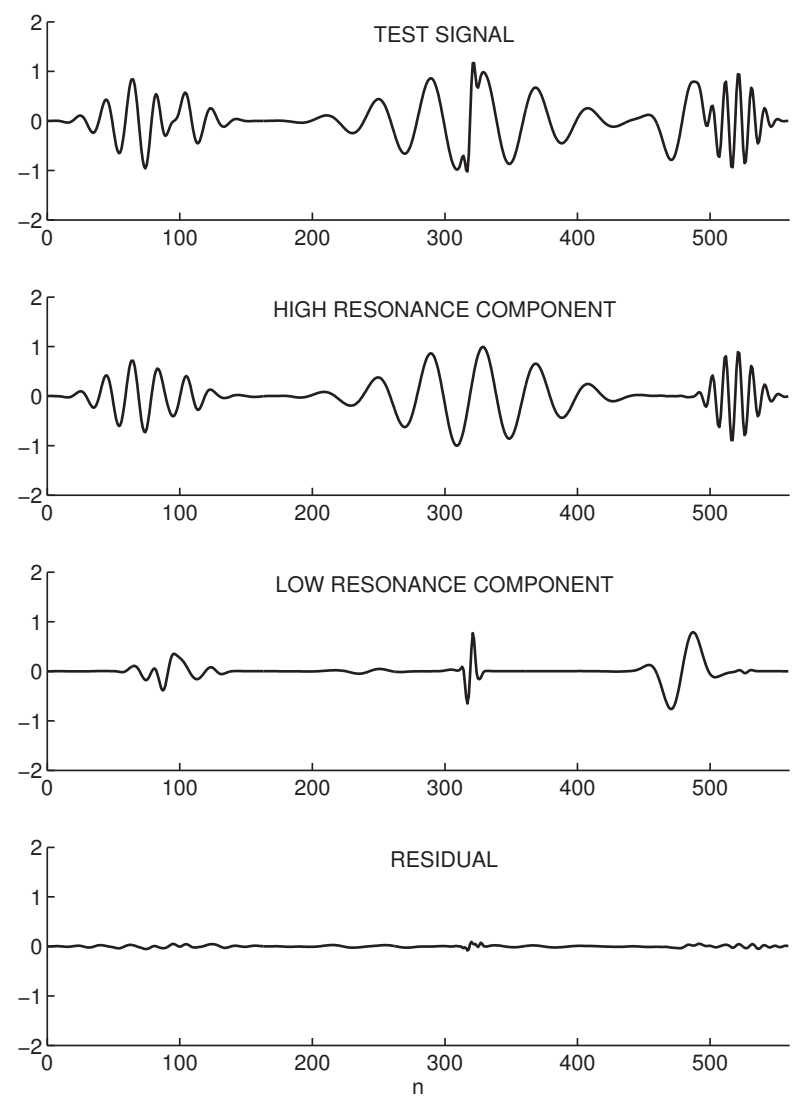

Fig. 2. Decomposition of a test signal into high and low resonance components. This result is obtained with 100 iterations of SALSA. The high resonance signal component is sparsely represented using a RADWT with high Q-factor. Similarly, the low resonance signal component is sparsely represented using a RADWT with low Q-factor.

[2] A. Beck and M. Teboulle. A fast iterative shrinkagethresholding algorithm for linear inverse problems. SIAM J. Imag. Sci., 2(1):183-202, 2009.

[3] J. M. Bioucas-Dias and M. A. T. Figueiredo. A new TwIST: Two-step iterative shrinkage/thresholding algorithms for image restoration. IEEE Trans. on Image Processing, 16(12):29923004, December 2007.

[4] V. Bruni and D. Vitulano. Transients detection in the time-scale domain. In Proc. 3rd International Conference on Image and Signal Processing (ICISP), pages 254-262, Berlin, Heidelberg, 2008. Springer-Verlag. LNCS 5099.

[5] I. Daubechies, M. Defriese, and C. De Mol. An iterative thresholding algorithm for linear inverse problems with a sparsity constraint. Commun. Pure Appl. Math, LVII:1413-1457, 2004.

[6] L. Daudet and B. Torrésani. Hybrid representations for audiophonic signal encoding. Signal Processing, 82(11):1595-1617, November 2002.

[7] M. E. Davies and L. Daudet. Sparse audio representations using the MCLT. Signal Processing, 86(3):457-470, March 2006.

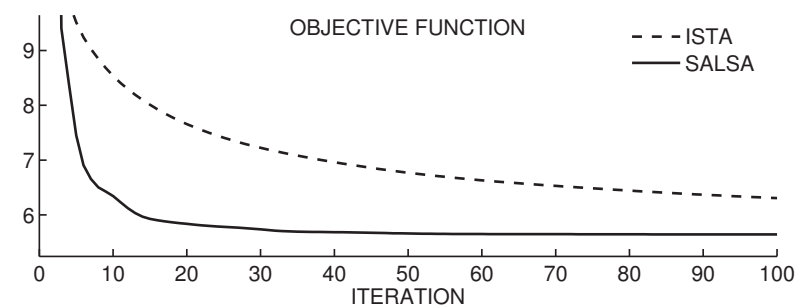

Fig. 3. Reduction of objective function during the first 100 iterations. SALSA converges faster than ISTA.

[8] M. Elad, B. Matalon, and M. Zibulevsky. Coordinate and subspace optimization methods for linear least squares with nonquadratic regularization. J. of Appl. and Comp. Harm. Analysis, 23:346-367, 2007.

[9] M. Elad, J. Starck, P. Querre, and D. Donoho. Simultaneous cartoon and texture image inpainting using morphological component analysis (MCA). J. of Appl. and Comp. Harm. Analysis, 19(3):340-358, November 2005.

[10] C. Févotte, L. Daudet, S. J. Godsill, and B. Torrésani. Sparse regression with structured priors: Application to audio denoising. In Proc. IEEE Int. Conf. Acoust., Speech, Signal Processing (ICASSP), volume 3, 2006.

[11] M. Figueiredo and R. Nowak. An EM algorithm for waveletbased image restoration. IEEE Trans. on Image Processing, 12(8):906-916, August 2003.

[12] M. A. T. Figueiredo, J. M. Bioucas-Dias, and M. V. Afonso. Fast frame-based image deconvolution using variable splitting and constrained optimization. In IEEE Workshop on Statistical Signal Processing, Aug. 31 - Sept 3 2009. See also http: //arxiv.org/abs/0904.4872v1.

[13] F. Jaillet and B. Torrésani. Time-frequency jigsaw puzzle: adaptive multiwindow and multilayered Gabor representations. Int. J. Wavelets Multires. Inf. Process., 5(2):293-316, March 2007.

[14] S. N. Levine and J. O. Smith III. A sines+transients+noise audio representation for data compression and time/pitch scale modications. In Proceedings of the 105th Audio Engineering Society Convention, 1998.

[15] S. Molla and B. Torrésani. An hybrid audio coding scheme using hidden Markov models of waveforms. J. of Appl. and Comp. Harm. Analysis, 18(2):137-166, 2005.

[16] I. W. Selesnick and I. Bayram. Oscillatory + transient signal decomposition using overcomplete rational-dilation wavelet transforms. In Proceedings of SPIE, volume 7446 (Wavelets XIII), 2009.

[17] J.-L. Starck, M. Elad, and D. Donoho. Image decomposition via the combination of sparse representation and a variational approach. IEEE Trans. on Image Processing, 14(10), 2005.

[18] J.-L. Starck, Y. Moudden, J. Bobina, M. Elad, and D.L. Donoho. Morphological component analysis. In Proceedings of SPIE, volume 5914 (Wavelets XI), 2005.

[19] S. J. Wright, R. D. Nowak, and M. A. T. Figueiredo. Sparse reconstruction by separable approximation. IEEE Trans. on Signal Processing, 57(7):2479-2493, July 2009. 